\title{
Morbidity in reflex sympathetic dystrophy
}

\author{
Clare S Murray, Alison Cohen, Tessa Perkins, Joyce E Davidson, John A Sills
}

\begin{abstract}
Reflex sympathetic dystrophy (RSD), an unusual diagnosis in general paediatrics, is well recognised by paediatric rheumatologists. This study reports the presentation and the clinical course of 46 patients (35 female, age range 8-15.2) with RSD. The patients saw professionals from an average of 2.3 specialties (range 1-5). Twenty five (54\%) had a history of trauma. Median time to diagnosis was 12 weeks (range 1-130). Many children had multiple investigations and treatments. Once diagnosis was made, treatment followed with physiotherapy and analgesics. Median time to recovery was seven weeks (range 1-140), with $27.5 \%$ relapsing. Nine children required assessment by the child and adolescent psychiatry team. This disease, though rare, has significant morbidity and it is therefore important to raise clinicians' awareness of RSD in childhood. Children with the condition may then be recognised and referred for appropriate management earlier, and spared unnecessary investigations and treatments which may exacerbate the condition.

(Arch Dis Child 2000;82:231-233)
\end{abstract}

Keywords: reflex sympathetic dystrophy; complex regional pain syndrome

Reflex sympathetic dystrophy, a disorder characterised by severe and continuous pain in an extremity associated with vasomotor instability, was first described by Mitchell in 1864 during the American Civil War. ${ }^{1}$ It is a well recognised diagnosis in adults but, although it occurs in children, is rarely mentioned in paediatric textbooks. Before 1978 only eight cases had been reported..$^{2-6}$ It is still probably underrecognised and underdiagnosed in children.

Since it was first described there has been much disagreement about the condition. It has been given a number of different titles, such as algodystrophy, Sudek's atrophy, causalgia, sympathetic maintained pain syndrome, and hand-shoulder syndrome. ${ }^{7}$ More recently, a revised taxonomic system for these disorders has been developed, and what we shall refer to in this paper as reflex sympathetic dystrophy (RSD) is now known as chronic regional pain syndrome type 1 (CRPS 1$){ }^{8}$

Since it was first described in paediatric patients, it has been realised that there are differences in presentation and treatment responses between adults and children. Generally, there is less often an antecedent history of trauma or surgery in children who develop RSD, and the outlook for recovery is better than in adults. ${ }^{9}$ Children rarely appear to develop trophic changes. ${ }^{10}{ }^{11}$ Psychological factors are thought to have a major role in the cause of this syndrome. ${ }^{12}$

The diverse symptomatology of RSD leads it to cross a wide spectrum of clinical disciplines. The resulting varied modes and routes of presentation may lead to a delay in diagnosis. Failure to recognise the condition may lead to unnecessary investigations and treatment, which may even exacerbate the condition.

We report the clinical course and treatment response of 46 children with RSD, presenting to a large children's hospital in the north west of England. This study aimed at determining the characteristics of the patients and their course to diagnosis. We postulated that the children with this disorder have a protracted course of illness, with significant morbidity, exacerbated by a delay in diagnosis.

\section{Methods}

The case notes of children who had presented to the paediatric rheumatology clinic, in the Royal Liverpool Children's Hospital, between 1 January 1990 and 31 December 1996, in whom a diagnosis of reflex sympathetic dystrophy was made, were reviewed. The diagnosis was made in all cases by a consultant paediatric rheumatologist by clinical evaluation, on the basis of the presence of a typical history; severe and continuous pain in an extremity, usually out of proportion to the inciting event (if any); and the presence of at least two descriptors of neuropathic pain and with some physical signs of autonomic dysfunction. The neuropathic pain descriptors were burning, dysaesthesia, paraesthesia, mechanical allodynia, and hyperalgesia to cold. The autonomic signs were cyanosis, oedema, mottling, coolness, altered perspiration, and changes in hair growth. Usually there was decreased movement of the affected limb and sometimes a bizarre posture was taken up.

The diagnosis of RSD was made in 46 patients during the study period. Once made, intensive physiotherapy was started, sometimes with the children as inpatients, by experienced paediatric physiotherapists. In addition, nonsteroidal anti-inflammatory drugs (NSAIDs) or paracetamol were sometimes used for analgesia. If a possible psychological problem was discovered, consultation with the child and adolescent psychiatry team was offered.

The children were followed up until symptoms had resolved and then reviewed three to six months later.

The case notes of the 46 children were reviewed and the patient's age, sex, and limb affected were recorded. Any precipitating event, their past and family history, and any psychological and family problems were noted. 
The number and nature of professionals previously seen with the problem, and the number and nature of investigations that were carried out in an effort to make the diagnosis, and treatments used before diagnosis, were recorded. The length of time taken to make the diagnosis was calculated from the onset of symptoms. Once diagnosis was made the length of time to recovery was calculated. Resolution was defined as complete resumption of age appropriate activity, including school attendance. Follow up continued for three to six months after resolution, and the number of children who relapsed during that time was identified.

\section{Results}

Reflex sympathetic dystrophy was diagnosed in 46 patients between 1 January 1990 and 31 December 1996 . Thirty five were girls $(76 \%)$ and 11 were boys $(24 \%)$, with a median age of 12.0 years (range $8-15.2$ ).

Fourteen $(30 \%)$ presented with an affected upper limb and $30(65 \%)$ with a lower limb affected. Two children presented with an upper and lower limb affected at the same time, both on the same side of the body.

Twenty six $(57 \%)$ children presented with a limb on the right side affected and 18 (39\%) children with a limb on the left side affected. Two children had limbs affected on both sides simultaneously.

Twenty five children attributed the problem to an episode of trauma. Seventeen of the children reported a history of joint problems, 11 of which appeared characteristic of RSD.

Eighteen $(39 \%)$ had a family history of joint problems, 11 in a first degree relative.

The children had consulted a number of professionals before referral to the paediatric rheumatology clinic. The average number consulted was 2.3 (range 1-5) different departments, including accident and emergency, general practice, orthopaedics, paediatrics, and adult rheumatology. Twenty five had attended accident and emergency, for a total of 37 consultations.

Before the diagnosis was made, all but three of the children had had some form of investigation; 42 (91\%) had had radiographs; $30(65 \%)$ blood tests; $17(37 \%)$ isotope bone scans; four $(9 \%)$ ultrasound scans; three $(7 \%)$ computed tomography; and six (13\%) magnetic resonance imaging; an average of 2.2 investigations per child.

Before the diagnosis 41 children had had some form of treatment. Twenty three had had physiotherapy and 21 had received NSAIDs. Seventeen of the 30 affected by lower limb RSD (57\%) had had a period of time non-weightbearing either by using crutches or in a wheelchair and seven $(23 \%)$ were put in plaster of Paris casts, five used "Tubigrip" supports. Eight $(57 \%)$ of the patients with an affected upper limb were treated with an arm sling. Two children had surgery.

The average time from onset of symptoms to diagnosis was almost six months (23.6 weeks), with a median of 12 weeks, range $1-130$ weeks. Once diagnosis was made all the patients received intensive physiotherapy, and 19 (41\%) received NSAIDs. Nine patients $(20 \%)$ consulted the child and adolescent psychiatry team, and a further two were offered consultation but refused.

After the diagnosis was made and treatment initiated, the time to recovery was calculated. Of the 46 children, three were lost to follow up, and three were continuing to have problems at the time the study was carried out (over 24,18, and 12 months from diagnosis). The median time to recovery was seven weeks from diagnosis (with a wide range from 1 to 140 weeks). During the follow up period 11 of the children who had made a full recovery relapsed.

\section{Discussion}

Reflex sympathetic dystrophy is less common in children than adults, but clinicians are becoming more aware of the problem. Our study of 46 children over a seven year period represented $3.9 \%$ of new referrals to our paediatric rheumatology clinic over that time.

We found that RSD was more common in girls than boys (3:1) and tended to occur during adolescence (median age 12 years). The lower limb was more often affected than the upper limb (2:1). The right side was only slightly more commonly affected than the left $(1.4: 1)$. Only just over half the children could recall an episode of trauma that might have been a precipitating event $(25 / 46(54 \%))$.

Reviewing their history $37 \%$ reported a previous joint problem - for example, irritable hip and chondromalacia patellae to episodes which, when described, appeared characteristic of previous RSD in $65 \%$ of the cases. Also, $39 \%$ of the children had a close family member with joint problems, such as rheumatoid arthritis, osteoarthritis, spondylitis, and sacroiliitis. Over half of these were in a first degree relative.

Interestingly, five of the 46 children had been admitted to hospital previously with abdominal pain. Three had required appendicectomies, all with a normal appendix on histology.

Our study confirms the major delay in diagnosis, which has been recognised previously. ${ }^{13}{ }^{14}$ Although the median time to diagnosis was 12 weeks, $15 \%$ took over 12 months to diagnose. Many patients saw a number of doctors before RSD was recognised. Often, many investigations for organic disease were carried out, attempting to explain an ill defined syndrome of pain and limited function. It is difficult to know retrospectively how many contributed positively to making the diagnosis. Many conditions had been excluded by the time the child had attended the paediatric rheumatology clinic, and no doubt these negative results were helpful at consultation. However, the diagnosis of RSD is made on clinical grounds, and limb radiography and bone scanning are probably the only investigations that may be helpful. Recent reports have observed increased, decreased, and normal radionucleotide uptake in children with RSD. ${ }^{15}$ These variations seem to depend on the duration of illness before 
scanning. The investigations are used early, and are helpful in the search for unrecognised organic disease.

We found that a significant number of children had had inappropriate treatments before the diagnosis was made. Over $50 \%$ of them had their limb immobilised, a treatment considered inappropriate in RSD, and which may even exacerbate the condition.

Once the diagnosis was made, intensive physiotherapy was started, sometimes with the children as inpatients. Many children also used NSAIDs or paracetamol. As other more invasive treatments such as sympathetic blockades have rarely been found to be effective in children, ${ }^{13}$ and their use in adults is now even in question, ${ }^{16}$ their use in our unit has generally been avoided. Physiotherapy management expertise in RSD may not be widely available and referral to a tertiary centre may be required. We often found a supportive relationship would develop between the physiotherapist and patient, and the child would disclose worries that had not been raised during other hospital consultations. This sometimes led to referral to the child and adolescent psychiatry team, which would not have happened otherwise.

Approximately $25 \%$ of the children were identified as having psychological issues that might have contributed to their disorder and were offered psychiatric assessment.

Overall, once diagnosis was made and intensive physiotherapy initiated, there was a good response to treatment. The median time to recovery was seven weeks. Twenty one per cent continued to have problems beyond three months and $10 \%$ beyond a year.

The relapse rate in our group was high, as has been shown before in other studies on this subject. ${ }^{17}$ Eleven of the 40 who made a full recovery, relapsed during the study period. Of these, about half occurred in the same limb. The high relapse rate, together with the number who seem to have had this condition previously, and the group with non-specific abdominal pain, all add weight to the already well established theory that psychological factors play an important part in the development of RSD in children. However, as with most chronic pain conditions it is difficult to determine the extent to which psychological factors cause or perpetuate the condition, or whether they themselves are a result of chronic pain. To date, prospective evaluation of patients with RSD has failed to show a statistically significant difference between patients and controls by a variety of psychological measures of individual and family functioning. ${ }^{18}$ As in other groups ${ }^{14}$ we found that many of the children were high achievers, either academically or in sport.

Although at the outset of the study we presumed that those who had a protracted course of illness would recover more slowly than those treated early, we could not confirm this in our group. Some children who had been diagnosed rapidly, within just a few weeks of onset, took months to recover, whereas others who had symptoms for months recovered in a matter of weeks once diagnosis and appropriate treatment were initiated.

Since carrying out our study, we have changed our management plan and automatically introduce the concept of referral to the child and adolescent psychiatry team. We have found families more accepting of their help when referral was made early, as a matter of routine, rather than waiting until patients failed to respond to physiotherapy alone.

In conclusion, RSD is a condition with a significant morbidity in childhood, causing temporary or permanent disability, and it is important for those working in paediatric rheumatology to raise their colleagues' awareness of the condition. Early recognition would prevent unnecessary investigations and treatments, which may exacerbate and prolong the condition. Although this study does not prove it, the prognosis for full recovery is still thought to be better in those treated early. Early recognition and subsequent appropriate treatment can surely therefore only help to decrease the morbidity in these children.

1 Mitchell SW, Morehouse GR, Kean WW. Gunshot wounds and other injuries of nerves. New York: Lippincott, 1864.

2 Fermaglich DR. Reflex sympathetic dystrophy in children. Pediatrics 1977;60:881-3.

3 Guntheroth WG, Chakmakjian S, Brena SC, et al. Posttraumatic sympathetic dystrophy: dissociation of pain and vasomotor changes. Am f Dis Child 1971;121:511-14.

4 Kozin F, McCarty DJ, Sims J, et al. The reflex sympathetic dystrophy syndrome. I. Clinical and histologic studies: evidence for bilaterality, response to corticosteroids and dence for bilaterality, response to corticosteroids
articular involvement. Am f Med 1976;60:321-31.

5 Stilz RJ, Carron H, Sanders DB. Reflex sympathetic dystrophy in a six-year-old: successful treatment by transcutanephy in a six-year-old: successful treatment by transcutan

6 Richlin DM, Carron H, Rowlingson JC, et al. Reflex sympaRichlin DM, Carron H, Rowlingson JC, et al. Reflex sympa-
thetic dystrophy: successful treatment by transcutaneous thetic dystrophy: successful treatment by

7 Gordon N. Reflex sympathetic dystrophy. Brain Dev 1996;18:257-62

8 Stanton-Hicks M, Janig W, Hassenbusch S, et al. Reflex sympathetic dystrophy: changing concepts and taxonomy. Pain 1995;63:127-33.

9 Bernstein BH, Singsen BH, Kent JT, et al. Reflex neurovascular dystrophy in childhood. F Pediatr 1978;93:211-15.

10 Kesler RW, Saulsbury FT, Miller LT, et al. Reflex sympathetic dystrophy in children: treatment with transcutaneous electric nerve stimulation. Pediatrics 1988;82:728-

Ruggeri SB, Athreya BH, Doughty R, et al. Reflex sympathetic dystrophy in children. Clin Orthop 1982;163: $225-30$

12 Sherry DD, Weisman R. Psychologic aspects of childhood reflex neurovascular dystrophy. Pediatrics 1988;81:572-8.

13 Stanton RP, Malcolm JR, Wesdock KA, et al. Reflex sympathetic dystrophy in children: an orthopedic perspective. Orthopedics 1993;16:773-80.

14 Wilder RT, Berde CB, Wolohan M, et al. Reflex sympathetic dystrophy in children. $\mathcal{F}$ Bone foint Surg Am 1992;74:91019.

15 Laxer RM, Allen RC, Malleson PN, et al. Technetium 99mmethylene diphosphonate bone scans in children with reflex neurovascular dystrophy. F Pediatr 1985;106:437-40.

16 Jadad AR, Carroll D, Glynn CJ, et al. Intravenous regional sympathetic blockade for pain relief in reflex sympathetic dystrophy: a systematic review and a randomized, double20.

17 Malleson PN, al-Matar M, Petty RE. Idiopathic musculoskeletal pain syndromes in children. $\mathcal{F}$ Rheumatol 1992; 19:1786-9.

18 Vieyra MA, Masek BJ, Berde C, et al. Children's pain and family functioning: a comparative analysis. $\mathcal{F}$ Pain Symptom Manage 1991;6:144. 